\title{
Self-organized 3D-printing Patterns Simulated by Cellular Automata*
}

\author{
Yasusi Kanada \\ Dasyn.com \\ yasusi@kanadas.com
}

\begin{abstract}
D printers are usually used for printing objects designed by 3D CAD exactly, i.e., deterministically. However, 3D printing process contains stochastic self-organization process that generate emergent patterns. A method for generating fully self-organized patterns using a fused deposition modeling (FDM) 3D printer with constant filament extrusion has been developed. By using this method, various patterns, such as stripes, splitting and/or merging patterns, and meshes can be generated. A cellular-automata-based computational model that can simulate such patterns have also been developed.
\end{abstract}

\section{Introduction}

3D-printing technologies, or additive manufacturing (AM) technologies [Gib 10], usually aim reproducing objects deterministically designed by using 3D computeraided design (CAD) tools. The object models created by CAD are horizontally sliced into thin "layers" by so-called "slicers", and a 3D printer prints the layers one by one. Especially, 3D printers for fused deposition modeling (FDM), such as those of Stratasys, Makerbot, or RepRap [Rep], shapes 3D objects by layering melted plastic filament extruded by a hot nozzle.

$3 \mathrm{D}$ printing process contains self-organization process that generates emergent and fluctuated patterns but they have been ignored. Printing processes contains bifurcations, and printing conditions and process including nozzle temperature, extrusion process, air motion, and so on, are fluctuated, so the generated patterns are partially self-organized and naturally randomized [Kan 14]. Although the printing process is usually controlled well so that self-organization is suppressed and the fluctuation usually does not cause serious problems to shape 3D objects, stochastic patterns caused by fluctuation can still often be seen in printed objects as described below. However, self-organized patterns generated by 3D printers are regarded as noises and are mostly ignored in 3D printing communities and industries.

Self-organized stochastic patterns can be seen in printed objects such as shown in Fig. 1. Two types of stochastic patterns can be seen in this photo. First, thin strings exist between standing edges. Although filament extrusion stops when the head is to move without extrusion, it is difficult to stop it completely and an unintended string is often generated. Second, small chunks of plastic exist at the end or center of strings in

*A longer version of this paper will be available as a chapter in "The 8th International Workshop on Natural Computing” published by Springer Verlag. 
Fig. 1. In contrast to strings, which are more uniform, the nozzle may create less uniform chunks. These and other stochastic patterns are explained more in a previous paper [Kan 14].

The emergence of FDM printing processes can be stressed by designing a fully selforganizing printing process that simulates onedimensional cellular automata (1D CA), which was proposed by the previous paper. This process generates emergent and stochastic 2D patterns by helical print-head motion. Basic patterns generated by this printing method are

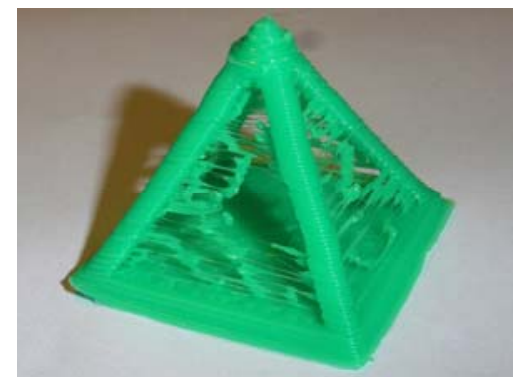

Fig. 1 Printed pyramids with strings and chunks (ABS, $38 \times 38 \times 33 \mathrm{~mm}$ ) stripes. However, stripes may sometimes spit or merge, waves may cross the stripes, and patterns may be meshes according to printing conditions. This study focuses on these types of patterns and shows a computational model based on 1D CA that can simulate them and suggest processes of other types.

The rest of this paper is organized as follows. Section 2 proposes a method for printing fully self-organized patterns and shows basic print results. Section 3 proposes a CA-based computational method to simulate the basic patterns. Section 4 shows various types of patterns, an extension to the CA-based method, and compares patterns generated by the printing and simulation methods. Section 5 describes the differences between the printing and simulation, and Section 6 concludes this paper.

\section{Method for Printing Fully Self-organized Patterns}

To generate 1D CA-like patterns, a 1D space without edges is used ([Wol 84] [Kan 94] etc.) in the method proposed by the previous paper [Kan 14]. The space occupied by the CA is topologically a circle. Therefore, to generate $1 \mathrm{D}$ patterns by an FDM printer, the print head can approximately draw circles in a clockwise or counterclockwise direction repeatedly and can extrude filament constantly (Fig. 2); however, a helix is used for the tool-path, i.e., the orbit of the print head, instead of layered circles because layer transitions create edges and spoil the pattern. In addition, because a head of a FDM 3Dprinter can only moves linearly, a "circle" is approximated by a collection of line segments. If the printing condition is selected carefully the printer can generate stochastic self-organized patterns.

The important conditions and parameters for this method, which are constant,

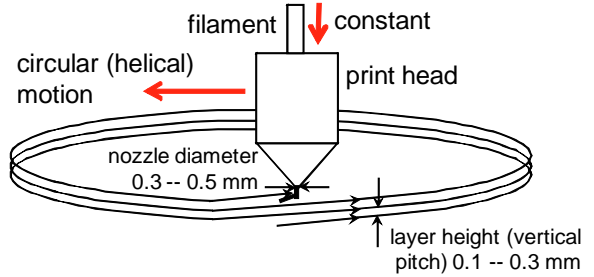

Fig. 2 1D pattern generation method are as follows. The nozzle diameter is usually 0.3 to $0.5 \mathrm{~mm}$, the average extruded filament cross-section $(c)$, which represents the velocity of extrusion, is much less than $0.2 \mathrm{~mm}^{2}(0.5 \phi)$, and the layer height $(h)$ is 0.1 to $0.3 \mathrm{~mm}$. The number of line segments in a "circle" is 72 . Other less sensitive parameters include the filament 


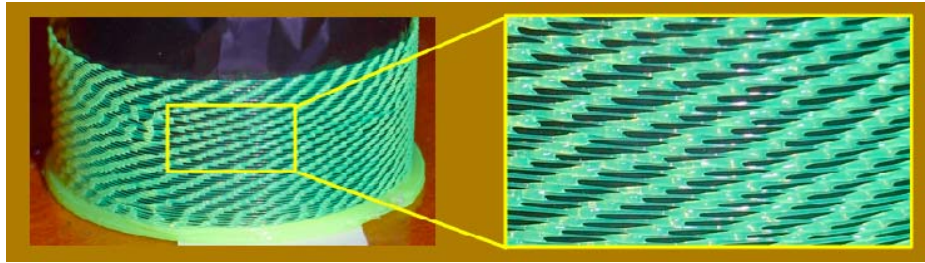

(a) Stripes with thick $(h=0.3 \mathrm{~mm})$

layers $(c=0.045)$

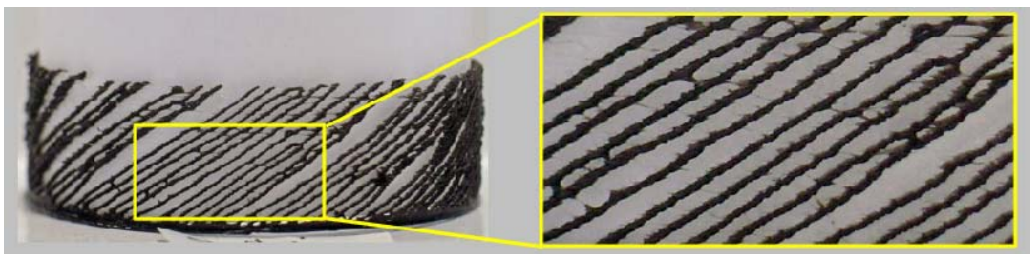

(b) Stripes with thin $(h=0.1$ $\mathrm{mm}$ ) layers $(c=0.02)$

Fig. 3 Typical printed patterns (by Rostock MAX)

material (usually ABS or PLA), the head temperature (220 to $260^{\circ} \mathrm{C}$ for $\mathrm{ABS}$ and 180 to $220^{\circ} \mathrm{C}$ for PLA), and the head motion velocity ( 40 to $150 \mathrm{~mm} / \mathrm{s}$ ).

The "layers" are formed by using the following method. The usually-used initial state is all one (i.e., filled); that means, the first layer of the circles is fully (and slowly) filled with plastic. The second and above layers are printed using the above parameters. In the second layer, filament sticks to the first layer mostly periodically because the fluctuation is still small. However, upper layers may be less periodically because more fluctuations are caused. An example of the printing process can be seen in YouTube [Das 13].

Although several examples of printed results are shown in the previous paper, typical patterns, which are shown in Fig. 3, are analyzed here at the first time. A Rostock MAX 3D-printer with a 0.5-mm nozzle and PLA filament was used for printing them. Fig. 3(a) shows skewed stripes and strings generated by clockwise (right to left) head motion with 0.3-mm layer height. Counterclockwise head motion creates stripes skewed toward the opposite direction. This figure shows that stripes are generated by stacking chunks and the strings connect stripes. Fig. 3(b) also shows stripes and strings, but the layer height is $0.1 \mathrm{~mm}$. The strings are very thin and mostly torn, so stripes are seldom connected by the strings.

\section{Basic Simulation Method and Results}

This section describes a computational model to simulate the self-organizing printing process, and shows relationships between printed patterns and simulation results.

\subsection{CA-based simulation method}

Figures 4 and 5 show a computational model and the whole algorithm for simulating the printed patterns. Figure 4 shows the model, which is based on 1D asynchronous 
CA [Ing 84][Hof 87][Wik a] (so the circle is quantized). This model simulates chunks but does not simulate strings. The values of cells, which grow upward, are calculated sequentially along the circle; that is, the value of one cell is decided in each step. Filament is constantly extruded in each step and it is accumulated until used for the cell (i.e., the

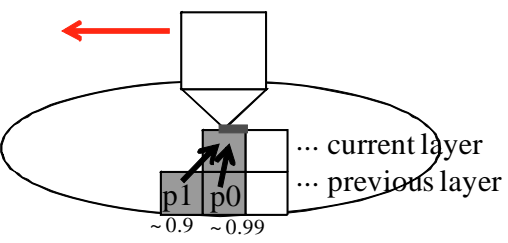

Fig. 4 Cellular automata for simulation cell value becomes 1). In the basic model, the accumulated filament is cleared when it is used; however, this rule may be varied. The value of the cell is probabilistically decided, and the filament is used only when it is sufficiently accumulated.

The value of a cell at layer $l$ and location $i$ (which is along the head motion) is defined by the following rule:

$$
\begin{aligned}
& \text { if cell }[l-1][i]=1 \text { then cell[}[l][i]=1 \text { at probability p0 } \\
& \text { else if cell[l-1][i+1] = } 1 \text { then cell[}[l][i]=1 \text { at probability p } 1 \\
& \text { else cell }[l][i]=0
\end{aligned}
$$

Here, cell $[l][i]$ means the value of cell at layer $l$ and $i$-th location on the circle. The value of each cell is on (1) or off (0). Chunk-stacking probabilities p0 and p1 represent the explicitly-introduced randomness and decide the lifecycle and directions of stripes. The whole algorithm is described in Fig. 5. Instead of using a two dimensional array, which was used in the previous paper [Kan 14], this algorithm uses a single-dimensional array for representing all the layers of cells because it is simpler and more convenient for simulating a helical motion.

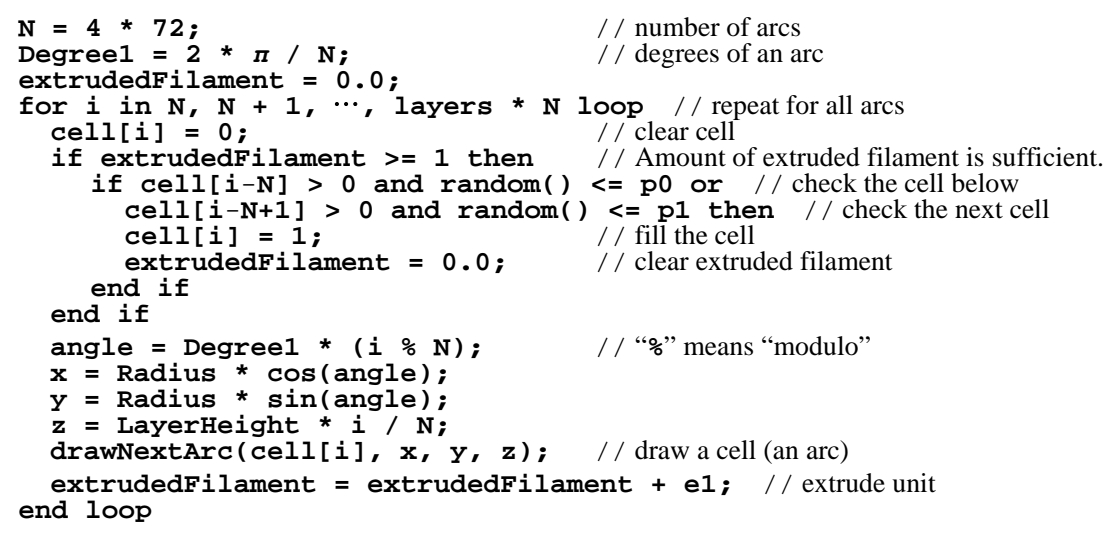

Fig. 5 Basic simulation algorithm

\subsection{Simulation of typical patterns}

Several results of simulating typical patterns using a program based on the algorithm are shown in Fig. 6. The simulation program that generates G-code, which is a type of computer-aided manufacturing (CAM) programs, was written by Python. The resulting G-code programs were visualized by a CAM tool called Repetier-Host. Several simulation results of typical patterns with stripes are shown in this figure. The 


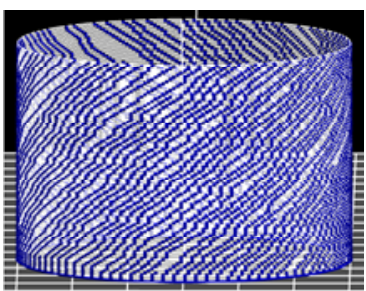

(a) Skewed stripes $(\mathbf{p} \mathbf{0}=$ 0.99, $\mathrm{p} 1=0.9$, e1 $=0.4$ )

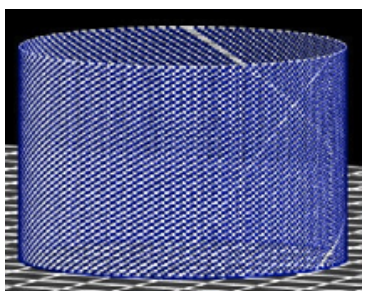

(b) Skewed stripes $(\mathrm{p} 0=$ $0.99, \mathbf{p 1}=\mathbf{1 . 0}, \mathrm{e} 1=0.4)$

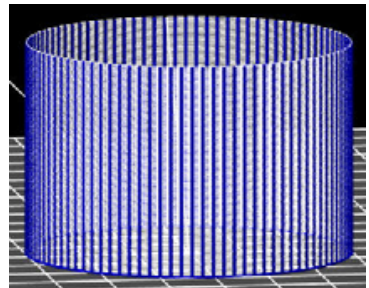

(c) Vertical stripes $(\mathbf{p 0}=\mathbf{1 . 0}$, $\mathrm{p} 1=0$ to $1, \mathrm{e} 1=0.4)$

Fig. 6 Simulation of stripes

value of $\mathrm{p} 0$ must be close to 1 to generate long-life patterns. If $\mathrm{p} 1$ is less than 1 , a noisy pattern such as shown in Fig. 6(a) is generated. If p1 is 1, a crisp stripes as shown in Fig. 6(b) is generated. If p0 equals to 1, a vertical stripes as shown in Fig. 6(c) is generated, but it is difficult to generate such a pattern by printing.

\section{Various Types of Patterns in Printing and Simulation}

Several types of printed patterns and simulation of the patterns are described in this section. The same printing parameter values as described in Section 2 were used unless otherwise stated.

\subsection{Extinction of stripes}

Chunks sometimes failed to stick to chunks below, so the stripes may be extinguished. It is difficult to see complete extinction in printed patterns because filament is constantly extruded. However, partial extinction is easily seen. An example is shown in Fig. 7(a). The circles show extinction of stripes.

Extinction patterns can be simulated by making $\mathrm{p} 0$ smaller in the simulation program. Figure 7(b) shows an extinction pattern with $\mathrm{p} 0=0.97$.

\subsection{Splitting and merging stripes}

Stripes are often split and merged. Fig. 8(a) shows a pure merging pattern and Fig. 8(b) contains both splitting and merging patterns. It is difficult to generate a pure

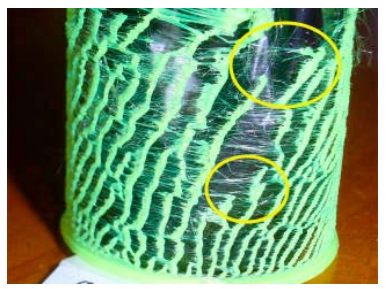

(a) Print result

(PLA,

$h=0.2$,

$c=0.02$, by

Printrbot Plus)

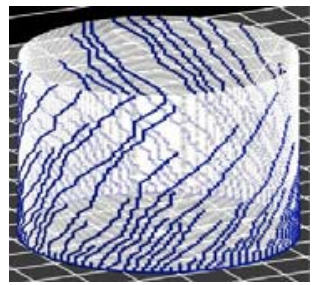

(b) Simulation

result

$(\mathrm{p} 0=0.97$,

$\mathrm{p} 1=0.9$,

$\mathrm{e} 1=0.6)$

Fig. 7 Extinction of stripes by printing and simulation 

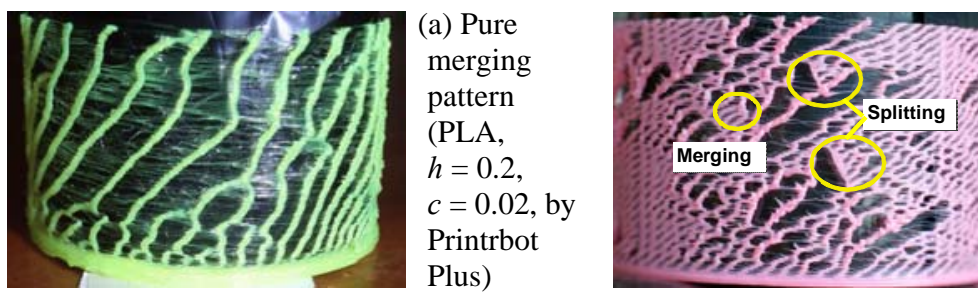

(b) Splitting and merging pattern

(ABS,

$h=0.25$,

$c=0.045$,

by Rostock MAX)

Fig. 8 Splitting and merging patterns by printing

splitting pattern. Vertical bars, which can be observed in Fig. 8(b), are caused by change of head velocity, which is caused by approximation of circles by linear lines.

The rule used for simulating splitting and merging patterns is described below and visualized in Fig. 9(a). The original computational rule is updated and two more parameters, $\mathrm{p} \_1$ and $\mathrm{C}(0<\mathrm{C}<1)$, are introduced because splitting and merging patterns cannot be simulated by the original algorithm.

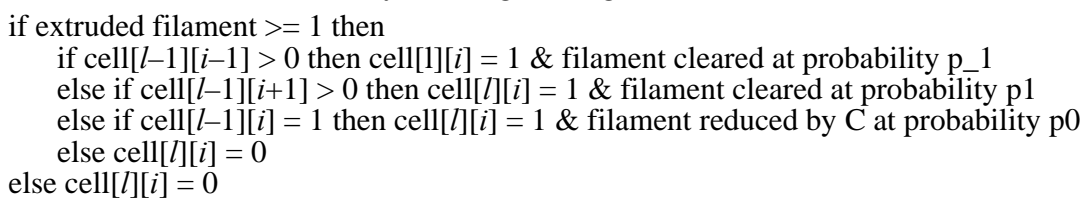

The two new parameters are used in the following way. First, this rule introduces a dependence between cell $[l][i]$ and cell[ $[-1][i-1]$, which enables splitting and which is controlled by a new chunk-stacking probability p_1. Second, the original rule always clears the extruded filament when it is used, but the new rule just subtract filament by $\mathrm{C}$ to preserve filament for splitting when the value of the cell below is 1 .

Figure 9(b) shows a simulation result that contains both splitting and merging. The above rule can generate splitting-and-merging patterns; however, because the above rule modification is not the only way of introducing such patterns and the generated patterns look differently from printed ones, a method for comparing the patterns and for evaluating the similarity should be developed, and the rule may have to be updated.

\subsection{Crossing waves}

Patterns that look like waves often seem to cross stripes. Typical waves can be seen in Fig. 10. In this figure, waves can be observed by changes of stripe angles and by thick

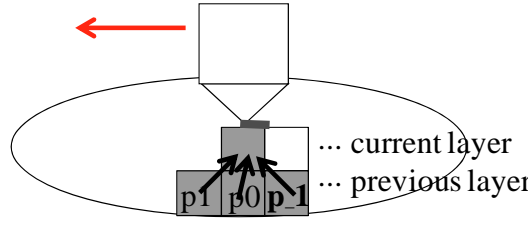

(a) Simulation method

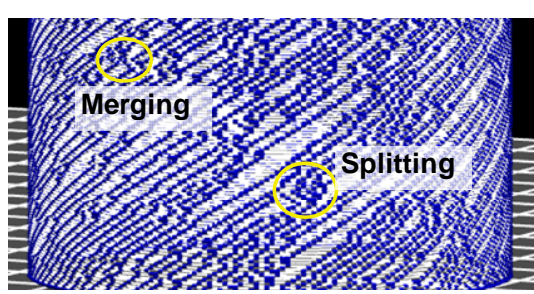

(b) Simulation result (p_1 $=0.7, \mathrm{C}=0.8$, $\mathrm{p} 1=0.9, \mathrm{p} 0=0.995, \mathrm{e} 1=0.4)$

Fig. 9 Extended simulation method for splitting and merging and simulation result 


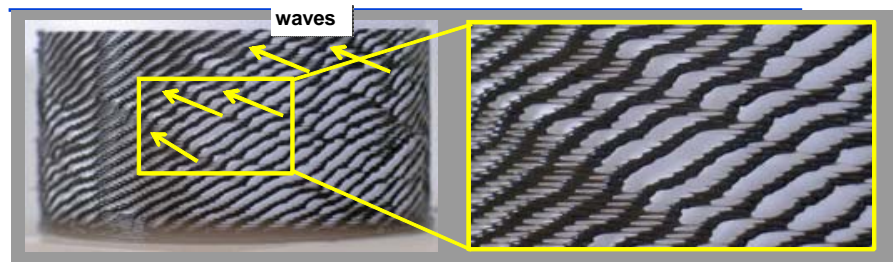

Fig. 10 Waves by printing ( $h=0.25, c=0.045$, by Rostock MAX)

strings or absence of strings.

It is not possible to simulate the waves in Fig. 10 exactly because the proposed algorithm does not simulate strings. However, waves are considered to be propagation of some change or noise and can widely be seen in patterns generated by the algorithm. Such waves can be seen easier by slightly modifying a crisp result. For example, if there is a defect in a vertical stripes shown in Fig. 6(c), it is propagated such as the results shown in Fig. 11(a) or (b). Figure 11(a) shows another modified simulation result generated by the layered-circle-based algorithm in the previous paper. In Fig. 11(b), zeros are randomly introduced to the initial layer (initial condition). Fig. 6(c) shows waves in a randomized simulation result. Vertical stripes are "propagated" nearly horizontally. Similar waves are also seen in Fig. 6(a).

\subsection{Meshes}

Stripes may sometimes be connected by layers of filaments such as shown in Fig. 12. Such patterns may be called "meshes". Meshes may be caused by waves; however, the crossing lines of filaments seem to be different from thick strings in patterns with waves such as shown in Fig. 10. Thickness of crossing lines depends on the velocity of extrusion. Meshes have not yet been successfully simulated by CA.

\section{Differences between Printing Process and Simplified Model}

The printed and simulated patterns are different in the following three points. First, the computational model only simulates chunks and does not simulate strings.

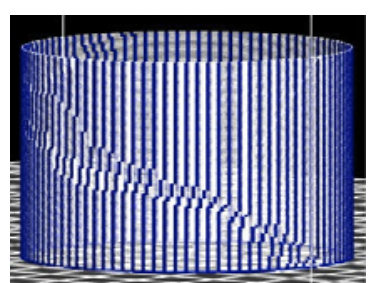

(a) Propagation of defect in layered-circle model

(p0 = 1.0, p1 = 0.4, e1 = 0.4)

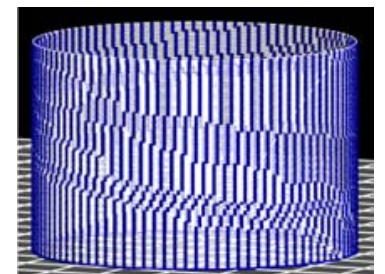

(b) Propagation of explicitly introduced noise

$(\mathrm{p} 0=1.0, \mathrm{p} 1=0.6, \mathrm{e} 1=0.4)$

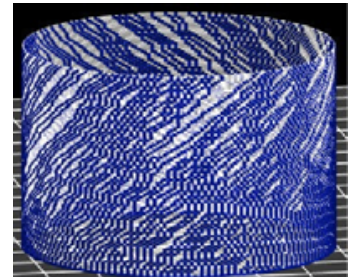

(c) Waves in randomized model

Fig. 11 Waves by simulation using the original algorithm 


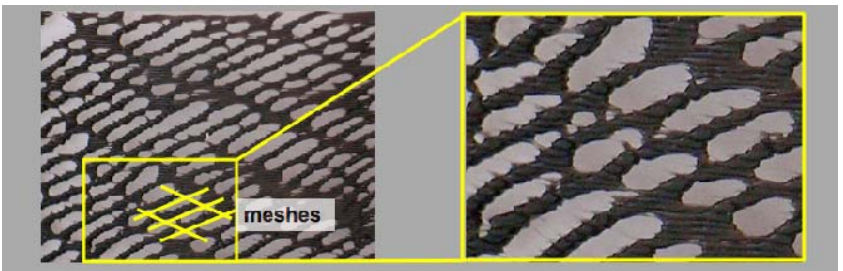

Fig. 12 Meshes by printing (PLA, $h=0.15, c=0.033$, by Rostock MAX)

Especially, patterns with waves (and probably meshes) thus cannot exactly be simulated. Second, the width of printed patterns (of radius direction) varies, but it is not simulated. If the width becomes larger, the number of active (1) cells becomes smaller even if the amount of extruded filament does not change. Third, printed stripes may bend or oscillate when the print head comes but such motions are not simulated. In addition, there may be more differences.

\section{Concluding Remarks}

FDM 3D-printers can generate self-organized and "naturally-randomized" patterns, which consist of chunks and strings. Fully self-organized patterns can be generated by the proposed printing method and various types of patterns, i.e., parallel stripes, splitting and merging stripes, waves, and meshes, can be generated by using this method. These types of patterns can be partially simulated by proposed 1D-CA-based computational method. However, there seem to be several differences between the printed and simulated patterns. In future work, these patterns should be compared by using a formal method and the CA-based model should be improved.

\section{References}

[Das 13] Dasyn.com, “Creating naturally-fluctuated patterns using a 3D printer”, YouTube, http://youtu.be/IJ15ysJR5l8

[Gib 10] Gibson, I., Rosen, D. W., and Stucker, B., “Additive Manufacturing Technologies”, Springer, 2010.

[Hof 87] Hofmann, M. I., "A Cellular Automaton Model Based on Cortical Physiology", Complex Systems, 1, pp. 187-202, 1987.

[Ing 84] Ingerson, T. E., and Buvel, R. L., "Structure in Asynchronous Cellular Automata", Physica D, Vol. 10, pp. 59-68, 1984.

[Kan 94] Kanada, Y., "The Effects of Randomness in Asynchronous 1D Cellular Automata", Artificial Life IV, 1994. (Unpublished extended version, http://www.kanadas.com/CA/AsyncCA/AsyncCAext.pdf)

[Kan 14] Kanada, Y., "3D Printing and Simulation of Naturally-randomized Cellularautomata”, 19th International Symposium on Artificial Life and Robotics (AROB 2014), January 2014.

[Rep] RepRap Wiki, http://reprap.org/

[Wik a] “Asynchronous cellular automaton”, Wikipedia, http://en.wikipedia.org/wiki/Asynchronous_cellular_automaton

[Wol 84] Wolfram, S., "Universality and Complexity in Cellular Automata", Physica D, Vol. 10, pp. 1-35, 1984. 